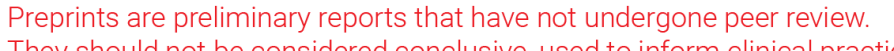 They should not be considered conclusive, used to inform clinical practice, or referenced by the media as validated information. \\ Radical Resection of T3N2M0 Esophageal Cancer Under Bilateral Thoracoscopy: A Case Report
}

\section{Mingcheng Gao}

Affiliated Hospital 6 of Nantong University https://orcid.org/0000-0002-7863-4180

Yulin Zhu

Affiliated Hospital 6 of Nantong University

Gu Yan

Affiliated Hospital of 6 Nantong University

Zhan Shi

Affiliated Hospital 6 of Nantong University

Huiwen Chang

Affiliated Hospital 6 of Nantong University

Jixiang Wu

Affiliated Hospital 6 of Nantong University

Jianxiang Song ( jxsongycsy@163.com )

Affiliated Hospital 6 of Nantong University https://orcid.org/0000-0003-4503-6464

\section{Case report}

Keywords: Esophageal cancer, Bilateral thoracic approach, Postoperative radiotherapy

Posted Date: February 9th, 2021

DOI: https://doi.org/10.21203/rs.3.rs-197945/v1

License: (c) (i) This work is licensed under a Creative Commons Attribution 4.0 International License.

Read Full License 


\section{Abstract}

Background: Many esophageal cancer patients with lymph node metastases during surgery are recommended for postoperative radiation therapy. However, postoperative radiation could cause many serious adverse reactions. Here, we introduce a new radical operation for esophageal cancer under bilateral thoracoscopy to reduce the adverse reactions of postoperative radiation.

Case presentation: A 50-year-old man was diagnosed with Esophageal squamous cell carcinoma by gastroscopy. Because the tumor was found to have obvious invasion and rapid pathology showed positive lymph nodes during the operation, which is in line with the postoperative chemotherapy indication, we adopted a bilateral thoracic approach for esophageal cancer to reduce adverse reactions of postoperative radiotherapy. We successfully guided the tubular stomach through the left thoracic aortic arch to the left thoracic cavity, and then performed an esophageal-tubular stomach anastomosis on the left neck under bilateral thoracoscopy to reduce radiation exposure to the remnant stomach. The operation went well.

Conclusion: For patients with esophageal cancer who are found to have radiotherapy indications during the operation, bilateral thoracoscopic radical resection of esophageal cancer should be preferred during the operation to improve the postoperative radiotherapy effect.

\section{Introduction}

Esophageal cancer (EC) is one of the most malignant tumor in China and surgery is still the cornerstone of the EC treatment [1,2]. However, surgical treatment alone has a high incidence of local recurrence and distant metastasis [3]. Therefore, neoadjuvant radiotherapy has become an increasingly common treatment for EC patients after surgery. While postoperative radiotherapy can improve overall survival (OS) and reduce local recurrence rate [4], the adverse reactions caused by radiotherapy, especially the occurrence of acute radiation gastritis, greatly decline the patients' life quality [5]. Here, we report a case of radical resection of esophageal cancer under bilateral thoracoscopy to reduce the adverse reactions caused by postoperative radiotherapy.

\section{Case Report}

A 50-year-old man presented to the hospital with difficulty swallowing. Physical examinations, including heart rate and bleed pressure, were normal. The gastroscopy tips showed an ulcerative esophageal tumor 34-38 cm from the upper incisors, and biopsy results indicated squamous cell carcinoma. Preoperative computed tomography (CT) showed囚thickening of the middle esophagus wall, consistent with malignant tumor (MT), and slight chronic inflammation of the right middle lung. During the operation, the patient underwent radical resection of esophageal cancer with bilateral thoracoscopic approach and the intraoperative pathological staging , according to the UICC-TNM classification (7th edition), indicated stage IIIB (pT3, pN2, cM0). 
Surgical method: First, the patient was placed in the prone position on the left side. Under $7 \mathrm{mmHg}$ pneumothorax pressure, a $30^{\circ}$ thoracoscopy was placed between the mid-posterior axillary line in the 7th intercostal space of the right chest. Then we took the subscapular line of the right posterior chest and two incisions at the mid-axillary line of the fourth intercostal space of the right chest as the main and auxiliary operation holes to free the thoracic esophagus. During the freeing process, we found that the esophageal mass was huge with extrapleural invasion and one of the irregularly enlarged lymph nodes invaded the thoracic aorta. Trial separation found it was frozen. Considering the risk of hemorrhage with forced separation, it was changed to local titanium clip marking for postoperative adjuvant radiotherapy QFigure 1囚. We cleared subcarinal nodes, thoracic paraesophageal lymph nodes and bilateral paratracheal lymph nodes Simultaneously. After the inventory was correct, a drainage tube was inserted, and the four holes of the right chest were closed and sutured one by one. The patient took the left side elevated position by $30^{\circ}$, re-sterilized the drape, cut off the anterior abdomen of the scapula hyoid muscle through an incision on the inner edge of the left cervical sternocleidomastoid muscle. Then we cut the scapula hyoid muscle anterior abdomen and free the cervical esophagus through an incision on the inner edge of the left cervical sternocleidomastoid muscle. Then we freed the cervical esophagus, broke and sutured the esophagus at the entrance to the thoracic cage of the neck. The umbilical incision was placed into a thoracoscopy. Four operation holes were made under the xiphoid process, bilateral costal arch and right upper abdomen to explore the chest cavity and free the stomach, and then the esophagus was pulled into the abdominal cavity. Take the midline incision of the upper abdomen to raise the gastric body, excise the cardia and part of the lesser curvature of the stomach, stretch the gastric body into a tube, and suture three stitches of the marking thread on the fundus of the stomach. The sixth intercostal space of the left thorax was inserted into the thoracoscope, and the anterior axillary incisions of the fourth and fifth intercostal spaces of the left thorax were taken as the operating holes (Figure 2). The silicone tube was guided under the laparoscope and pulled from the left thoracic aortic arch into the abdominal cavity through the mediastinum through the esophageal hiatus. After connecting with the tubular stomach marking line, guide the tubular stomach through the left thoracic aortic arch to the left neck, and perform an esophageal-tubular stomach anastomosis on the left neck (Figure 3a,b). During the operation, an anesthesiologist was asked to insert the duodenal nutrition tube and gastrointestinal decompression tube from the nasal cavity and suture the incision layer by layer.

\section{Discussion And Conclusion}

According to the latest 2020 guidelines for the diagnosis and treatment of esophageal cancer issued by the Chinese Society of Clinical Oncology (CSCO), postoperative radiotherapy is recommended for patients whose pathological stage reaches $\mathrm{T} 4$ or lymph node positive after radical resection of esophageal cancer. Since the conventional minimally invasive esophagectomy (MIE) procedure raises the tubular stomach to the posterior mediastinal position, the tubular stomach will be exposed to the target area of the original esophageal bed lymphatic drainage area during postoperative radiotherapy and the incidence of thoracic radiation gastritis will be increased [6]. Under the action of radiation, the gastric mucosa can be congested and edema in mild cases, endarteritis obliterans and fibrosis can occur in severe cases, and 
complications such as erosion, ulcers, bleeding, and perforation may occur in the later period [7]. Many studies have shown the position of the thoracic stomach is related to the occurrence of radiation thoracic gastritis [7-10]. Song Chunyang et al. [11] studied 104 patients accepting intensity-modulated radiotherapy (IMRT) after EC surgery and divided patients into three groups: left thoracic stomach group, right thoracic stomach group and posterior mediastinal stomach group. The results showed the incidence of radiation thoracic gastritis in left thoracic is much lower than that of posterior mediastinal. We adopt a bilateral thoracoscopic approach to draw the tubular stomach to the left thoracic cavity through the left thoracic approach, and then anastomosis with the cervical esophagus, which not only achieves the effect of minimally invasive treatment, but also keeps the tubular stomach away from the target area of the original esophageal bed lymphatic drainage area. This reduces the possibility of acute thoracic radiation gastritis, and it also makes the target area of the original esophageal bed lymphatic drainage better exposed, effectively ensuring the effect of radiotherapy.

Therefore, for patients with obvious tumor invasion during the operation, residual possibility or rapid pathology prompting positive lymph nodes and other postoperative radiotherapy indications, this type of surgery should be preferred.

\section{Abbreviations}

EC: Esophageal cancer; CT: Computed tomography; MT: Malignant tumor; CSCO: Chinese Society of Clinical Oncology; MIE: Minimally invasive esophagectomy; IMRT: intensity-modulated radiotherapy

\section{Declarations}

Acknowledgements

Not applicable.

Funding

None.

Availability of data and materials

All available data are presented in the case.

Authors' contributions

JS designed the research. MG, $Y Z$, and $Y G$ have been involved in the management of the patient and in the conception of the manuscript. ZS, JW and HC performed pathological diagnosis and surgical treatment for the patient. $M G$ has been involved in the drafting of the manuscript and its critical revision for important intellectual content. All authors read and approved the final manuscript. 
Ethics approval and consent to participate

Not applicable.

Consent for publication

Written informed consent was obtained from the patient for publication of this case report and accompanying images. A copy of the written consent is available for review by the Editor-in-Chief of the journal.

\section{Competing interests}

The authors declare that they have no competing interests.

Author details

${ }^{1}$ Department of Cardiothoracic Surgery, Affiliated Hospital 6 of Nantong University, 2 Xindu West Road, Jiangsu 224300, People's Republic of China

\section{References}

1. Gisbertz SS, Hagens ERC, Ruurda JP, Schneider PM, Tan LJ, Domrachev SA, et al. The evolution of surgical approach for esophageal cancer. Ann N Y Acad Sci. 2018;1434(1):149-55.

2. Chen J, Pan J, Liu J, Li J, Zhu K, Zheng X, et al. Postoperative radiation therapy with or without concurrent chemotherapy for node-positive thoracic esophageal squamous cell carcinoma. Int $J$ Radiat Oncol Biol Phys. 2013;86(4):671-7.

3. Schreiber D, Rineer J, Vongtama D, Wortham A, Han P, Schwartz D, et al. Impact of postoperative radiation after esophagectomy for esophageal cancer. Journal of thoracic oncology : official publication of the International Association for the Study of Lung Cancer. 2010;5(2):244-50.

4. Lin H-N, Chen L-Q, Shang Q-X, Yuan Y, Yang Y-S. A meta-analysis on surgery with or without postoperative radiotherapy to treat squamous cell esophageal carcinoma. Int J Surg. 2020;80:18491.

5. Shen W-B, Wang Y-F, Gao H-M, Zhu S-C, Li Y-M, Li S-G, et al. Dosimetric Predictors of Radiation Gastritis Due to Postoperative Intensity Modulated Irradiation Therapy in Patients with Esophageal Squamous Cell Carcinoma After Radical Esophagectomy. Cancer Biother Radiopharm. 2019;34(7):419-26.

6. Shen Wenbin, Gao Hongmei, Cao Yankun, Zhu Shuchai, Li Youmei, Li Shuguang, et al. The dose study of radiation gastritis caused by esophageal cancer radical postoperative prophylactic irradiation. Chin J Radiat Oncol. 2016;25(8):818-22.

7. Monma S, Kato K, Shouji H, Okita N, Takashima A, Honma Y, et al. Gastric mucosal injury and hemorrhage after definitive chemoradiotherapy for locally advanced esophageal cancer. Esophagus : 
official journal of the Japan Esophageal Society. 2019;16(4):402-7.

8. Kavanagh BD, Pan CC, Dawson LA, Das SK, Li XA, Ten Haken RK, et al. Radiation dose-volume effects in the stomach and small bowel. Int J Radiat Oncol Biol Phys. 2010;76(3 Suppl):S101-S7.

9. Chen J, Pan J, Zheng X, Zhu K, Li J, Chen M, et al. Number and location of positive nodes, postoperative radiotherapy, and survival after esophagectomy with three-field lymph node dissection for thoracic esophageal squamous cell carcinoma. Int J Radiat Oncol Biol Phys. 2012;82(1):475-82.

10. Adelstein DJ, Rice TW, Rybicki LA, Saxton JP, Videtic GMM, Murthy SC, et al. Mature results from a phase II trial of postoperative concurrent chemoradiotherapy for poor prognosis cancer of the esophagus and gastroesophageal junction. Journal of thoracic oncology : official publication of the International Association for the Study of Lung Cancer. 2009;4(10):1264-9.

11. Song Chunyang, Zhu Shuchai, Shen Wenbin, Su Jingwei, Gaosna, Zhao Yan, et al. The relationship between the dose-volume indexes and acute toxicity of intrathoracic stomach in esophageal cancer patients receiving radiotherapy after esophagectomy. Chinese Journal of Radiological Medicine and Protection. 2020;40(2):106-11.

\section{Figures}

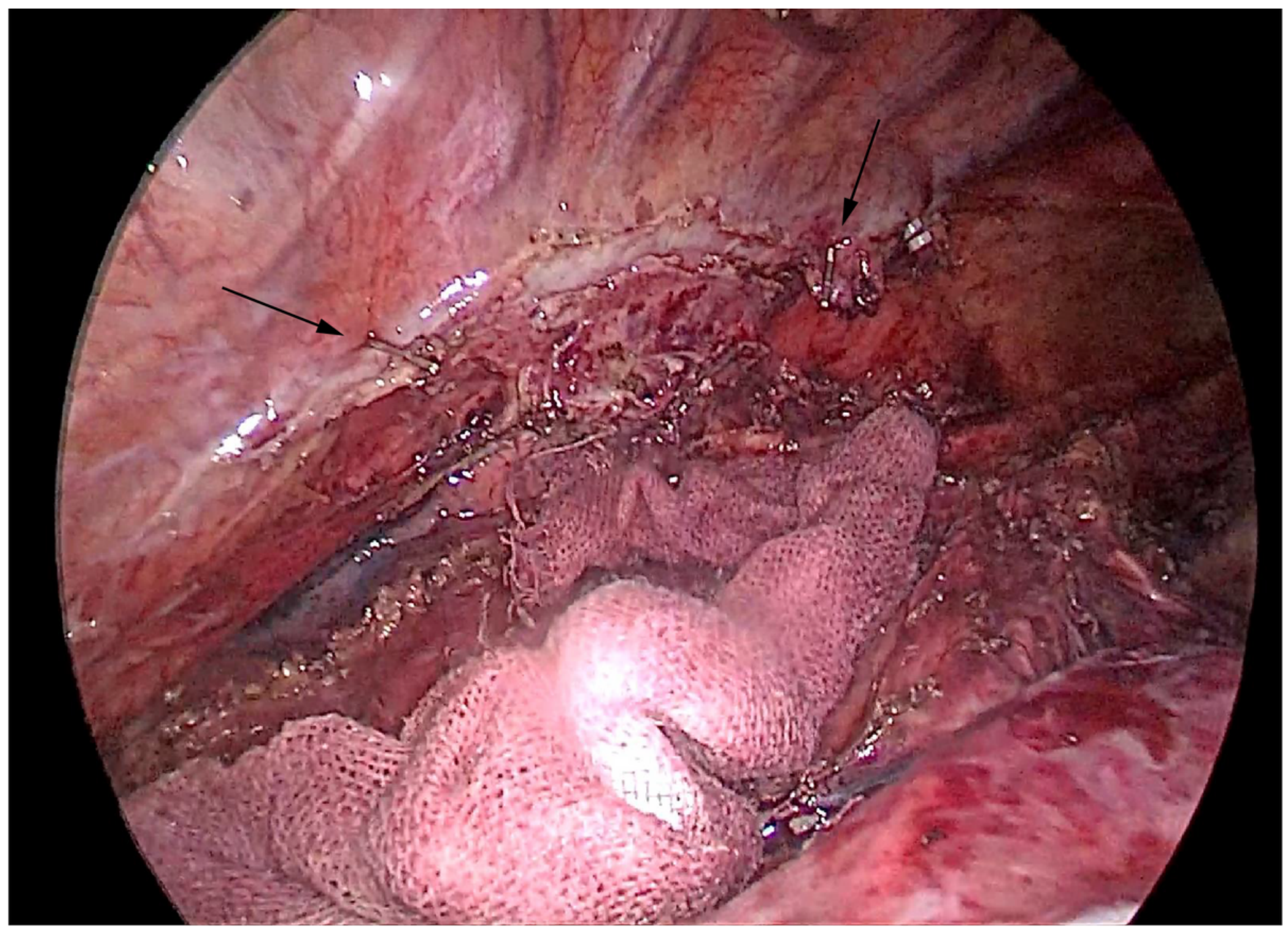




\section{Figure 1}

Enlarged lymph nodes invade the thoracic aorta, marked by titanium clips (arrow)

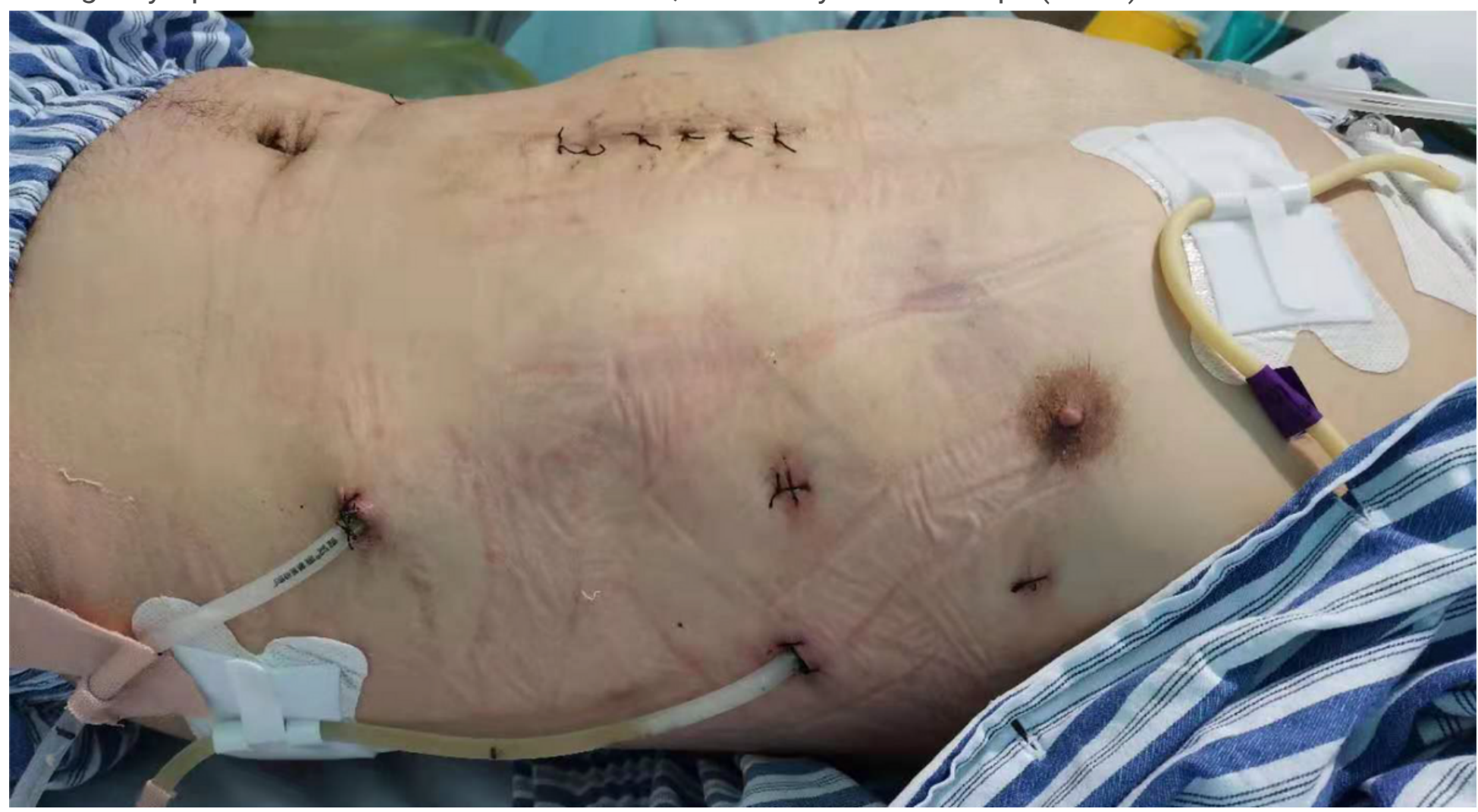

\section{Figure 2}

We placement three access ports (4th, 5th and 6th intercostal spaces) for the left thoracic procedure
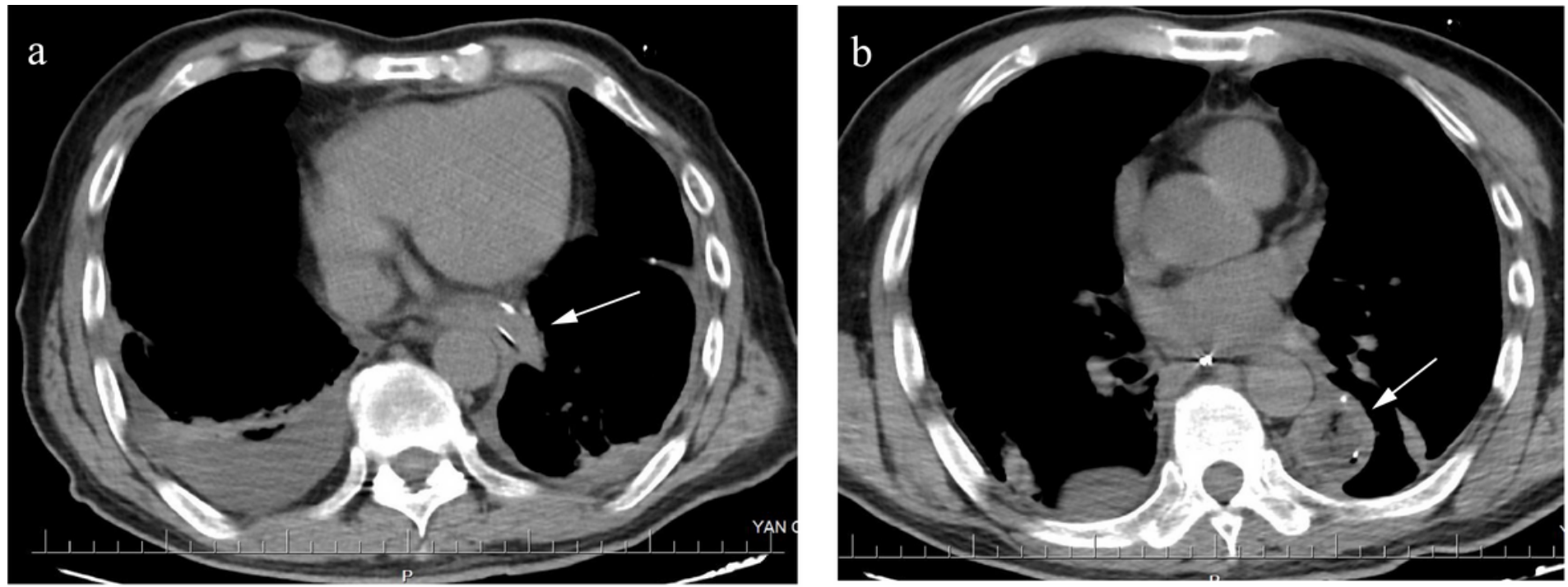

\section{Figure 3}

Tubular stomach (arrow) through the left thoracic aortic arch to the left chest cavity 